\title{
Analysis of simple supported plate for active vibration control with piezoelectric sensors and actuators
}

\author{
Kapil Narwal $^{1 *}$, Deepak Chhabra ${ }^{2}$ \\ ${ }^{1 *}$ (University Institute of Engineering and Technology, Maharshi Dayanand University, India) \\ ${ }^{2}$ (University Institute of Engineering and Technology, Maharshi Dayanand University, India)
}

\begin{abstract}
This work is aimed at the active vibration control of a flexible structures using piezoelectric material. A simple supported plate structure, which is supported at two opposite ends, is taken as the flexible structure with piezoelectric materials as sensors and actuators. Sensors and actuators, which are square in shapes, are embedded to the parent structure. The active controller was designed to control first three modes of vibration of plate. First, the analysis for the transient vibrations in a simple supported plate structure was performed which was followed by the analysis of the same simple supported plate structure when embedded upon with a pair of sensor and actuator. The plate was discretised into several small rectangular elements $(6 X 6,7 X 7 \ldots 11 X 11)$ of identical size in order to assign the different locations to sensors and actuators, which were assumed to be of the exact shape of the discretized plate elements. Further a LQR controller is applied for attenuating the global structural vibration. Settling time for each different location of piezoelectric patch location was observed which was then followed by an interpretation for the optimal location for piezoelectric patch for maximizing the vibration control. The model designed for study was duly verified with the results from past literature and an agreement between the both was observed.
\end{abstract}

Keywords -Active Vibration Control, Plate, Vibration Control, Smart Structure, LQR, POF

\section{INTRODUCTION}

A Smart Structures is the structure that can produce some responses to the external disturbances and actively control the same in real time to maintain the mission requirements. The smart structure essentially consists of a host structure with sensors and actuators which are intelligently coordinated by a controller. Self- diagnosis and adaptability to the environmental variations make this integrated system a smart structure. Over the last few years the technology of Smart Materials and Structures and in specific Piezoelectric Smart Structures has grown up considerably which widely found its application in suppressing the unwanted structural vibrations. The present document attempts analysis of a simple supported plate for Active Vibration control using piezoelectric material as sensors and actuators in the form of patches. An exhaustive literature has been reviewed and analysed for having reached at consensus on what has been done so far and highlighting where there are the scopes available for further improvement. Researchers have studied the behavioural aspects of piezolaminated plates. Using piezoelectric smart structures for the active vibration control has paid considerable attention in the last decade. Balamurugan V. and Narayanan S. [3] studied the mechanics for the coupled analysis of piezolaminated plate and curvilinear shell structures and their vibration control performance. A plate/shell structure with thin PZT layers embedded on top and bottom surfaces is considered. Active vibration control performance of plates and shells with distributed piezoelectric sensors and actuators have been studied. Caruso G. et al. [8] studied the vibration control of an elastic plate, clamped along one side and excited by impulsive transversal force acting in correspondence of a free corner. A modal model obtained by employing a suitable finite-element formulation together with a modal reduction, was used in the controller design. Tylikowski A. [5] analysed the capacitive shunting distributed piezoelectric elements perfectly glued to the vibrating annular plate excited by harmonic displacement of the inner plate edge. The equations of piezoelement were coupled with the equations of plate motion by the surface strain terms.

Different finite element methods have been proposed by researches for the modelling of piezolaminated plates. Lam K. Y. et al. [1] developed a finite-element model based on the classical laminated plate theory for the active vibration control of a composite plate containing distributed piezoelectric sensors and actuators from the variation principle. A negative velocity feedback control algorithm coupling the direct and converse piezoelectric effects was used. Verification of the proposed model was on a cantilever composite plate. Narayanan S. and Balamurugan V. [10] studied the finite element modelling of laminated structures with distributed piezoelectric sensor and actuator layers. Beam, plate and shell type elements have 
been developed incorporating the stiffness, mass and electromechanical coupling effects of the piezoelectric laminates. Lin J. C. and Nien M.H. [15] discussed the adaptive modelling and shape control of laminate plates with piezoelectric actuators. A finite element formulation was developed for dynamic and static response of laminated plates. A composite plate with different location of mechanical load was studied analytically and experimentally.

Studies on active vibration controlling capabilities of these plates have been done. Yaman Y. et al. [7] presented the theoretical and experimental results of the modelling of a smart plate for active vibration control. The smart plate consists of a rectangular aluminum plate modelled in cantilever configuration with surface bonded piezoelectric patches. The patches are symmetrically bonded on top and bottom surfaces. The study used ANSYS(v.5.6) software to derive the finite element model of the smart plate. The optimal sensor locations were found and actual smart plate was produced. Mukherjee A. et al. [6] presented the active vibration control of stiffened plates. A stiffened plate finite element with piezoelectric effects was formulated. A velocity feedback algorithm was employed. Numerical examples for vibration control of isotropic and orthotropic stiffened plates were presented. Costa L. et al. [14] derived a reduced model for a piezoelectric plate and to study its actuator and sensor capabilities. Study on the actuator and sensor capabilities of this model was done. Two discrete non-differentiable multi-objective optimization problems were used, which were solved by genetic algorithms.

Optimal design techniques have been worked out for the piezolaminated plates. Li Y. et al. [4] formulated a new optimal design methodology for the placement of piezoelectric actuator and the feedback gains in vibration suppression of flexible structure. The effect of changes in the mass and the stiffness of the structure combined with control performance index lead to solutions that were independent of initial conditions of the flexible structure. Qiu Z. C. et al. [17] used piezoelectric ceramics patches as sensors and actuators to suppress the vibration of the smart flexible clamped plate. A method for optimal placement of piezoelectric actuators and sensors on a cantilever plate was developed. An experimental setup of piezoelectric smart plate was built up. Yang Y. and Zhang L. [13] studied a simply supported rectangular plate subject to in-plane forces, resting on an elastic foundation and excited by a PZT actuator. The optimal placement of the PZT actuator discussed. A general procedure to determine the optimal excitation locations was proposed.

Several controllers have been proposed by researchers for the active control of vibration using piezoelectric materials on plates. Pai P. F. et al. [2] investigated non-linear saturation control, non-linear internal resonance control, and linear position-feedback control of steady-state and transient vibrations of a cantilever beam by using PZT patches. To test this SIMULINK software was used. Both numerical and experimental results proved efficient. Li Y.Y. et al. [9] analysed the design of 1-synthesis controller for vibration control of a plate with piezoelectric patches. A numerical model was derived and a controller was synthesized. Experiments were then performed with MATLAB/Simulink.Shimon P. et al. [11] developed an efficient controller for vibration reduction in a fully clamped plate and an investigation between control methodologies and actuators was done. Theoretical and experimental studies were undertaken with verifying results. Luo Q. and Tong L. [12] presented a high precision control for the specified plate twisting and bending shapes using the orthotropic piezoelectric actuators. Finite element analysis for composite plates with the adhesively bonded piezoelectric stiffeners was derived and verified on NASTRAN. Shirazi A. H. N. et al. [19] investigated the active vibration control of a simply supported rectangular plate with fuzzy logic control and compared to the results obtained with the application of PID control. Differential equation of motion for a rectangular plate made of functionally graded material equipped with piezoelectric patches is derived. A comparison is made between the application of PID control and FLC to dampen the plate vibration. Kang $\mathrm{Z}$ and Tong L. [18] investigated the optimal spatial distribution of single-channel actuation voltage in static structural shape control problem. The Mindlin plate theory was used. A problem was transformed into a continuous one and a power-law function relating the design variables and the applied voltages was proposed. Nguyen Q. et al. [16] presented a new evolutionary algorithm to solve various structural shape control problems of smart composite plate structures with active piezoelectric actuators. The linear least square method and the features of evolutionary strategies were employed. The evolutionary piezoelectric actuator design optimization was proposed. Several numerical examples were presented for verification. 


\subsection{Finite Element Formulation}

\section{METHODOLOGY}

An isotropic elastic rectangular plate of homogenous material is considered with its dimensions length $\mathrm{L}$, breadth $\mathrm{B}$, and thickness $\mathrm{H}$. then the plate is discretized into some finite number of smaller elements of identical shapes and sizes. Considering $\mathrm{M}$ is the number of elements along the length of the plate and $\mathrm{N}$ are the number of elements along the breadth. Each element is considered to be rectangular in shape with nodes $\mathrm{i}, \mathrm{j}, \mathrm{l}$ and $\mathrm{m}$; and with dimensions length $2 \mathrm{a}$ and breadth $2 \mathrm{~b}$ and thickness $\mathrm{h}$.

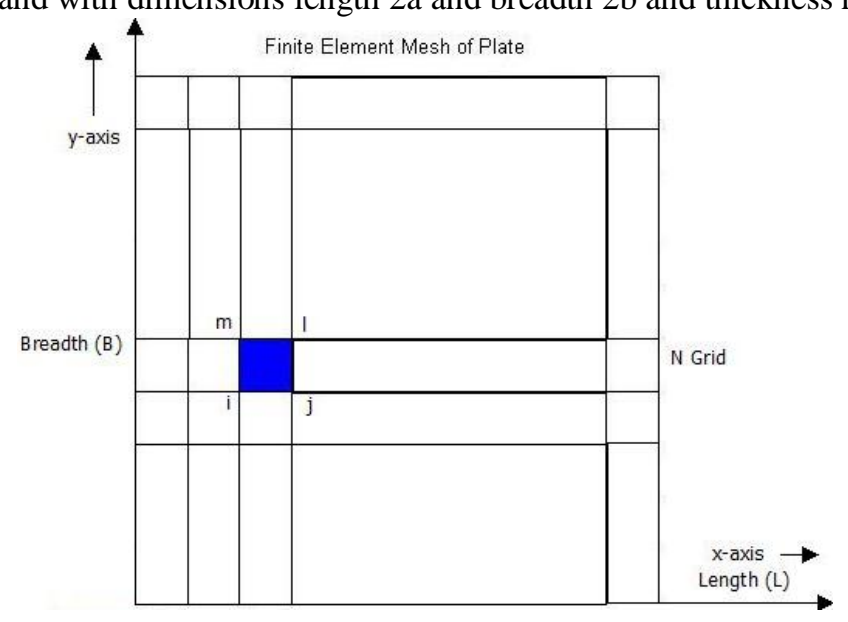

Considering all three degree of freedom for each node viz. $w, \theta_{x}$ and $\theta_{y}$ where $w$ is the transverse, $\theta_{x}$ and $\theta_{y}$ are the rotational degree of freedom in $x$ and $y$ directions respectively. The local coordinates therefore are $\xi=x / a$ and $\eta=y / b$ which lie at the center of element. The transverse displacement of element is:

$w=\left[N_{i}(\xi, \eta) N_{j}(\xi, \eta) N_{l}(\xi, \eta) N_{m}(\xi, \eta)\right]\{u\}_{E}$

Where $\{u\}_{E}=\left(w_{i} \theta_{x i} \theta_{y i} \ldots \ldots \ldots . . w_{m} \theta_{x m} \theta_{y m}\right)^{T}$

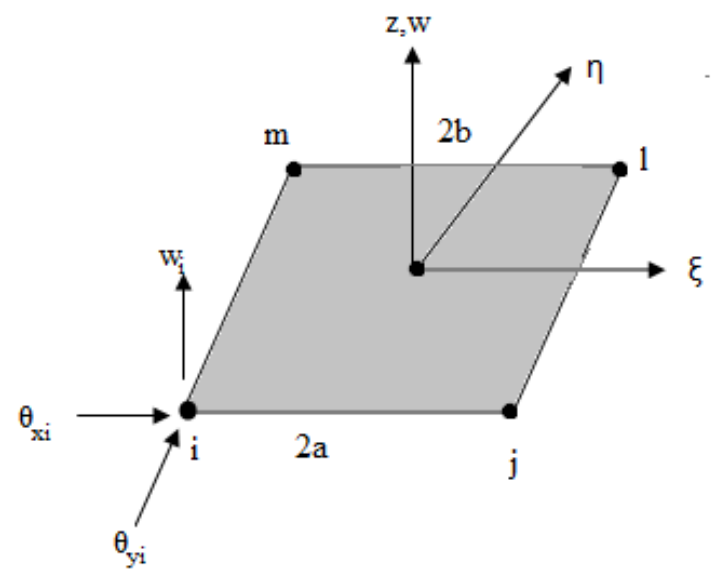

Where $w_{k}$ being the transverse displacement at node $k, \theta_{x k}$ and $\theta_{v k}$ are the rotational displacements about $x$-axis and y-axis respectively. $\theta_{x k} \& \theta_{y k}$ are given by

$\theta_{x}=\frac{d w}{d y}, \theta_{y}=\frac{-d w}{d x}$

The shape function elements at node $k$ are given by 


$$
N_{k}^{T}(\xi, \eta)=\frac{1}{8}\left[\begin{array}{l}
\left(1+\xi_{k} \xi\right)\left(1+\eta_{k} \eta\right)\left(2+\xi_{k} \xi+\eta_{k} \eta-\xi^{2}-\eta^{2}\right) \\
b\left(1+\xi_{k} \xi\right)\left(\eta_{k}+\eta\right)\left(\eta^{2}-1\right) \\
-a\left(\xi_{k}+\xi\right)\left(\xi^{2}-1\right)\left(1+\eta_{k} \eta\right)
\end{array}\right]
$$

Where $k=i, j, l, m$;

$\xi_{k}, \eta_{k}$ are the co-ordinates of a node $k$ (taking $\xi_{k}=(-1,+1)$ and $\eta_{k}=(-1,+1)$ ). There exists three degrees of freedom for each node $w, \theta_{x}, \theta_{y}$ and as each element has four nodes so twelve degrees of freedom. The kinetic energy and strain energy of element are

$$
\begin{aligned}
& T_{E}=\frac{1}{2}\{\dot{u}\}_{E}^{T}[m]_{E}\{\dot{u}\}_{E} \\
& U_{E}=\frac{1}{2}\{\dot{u}\}_{E}^{T}[k]_{E}\{\dot{u}\}_{E}
\end{aligned}
$$

The element mass matrix $[m]_{E}$ and element stiffness matrix $[k]_{E}$ are given as [2]

$$
\begin{aligned}
& {[m]_{E}=\rho a b \int_{-1}^{1} \int_{-1}^{1}[N(\xi, \eta)]^{T}[N(\xi, \eta)] d \xi d \eta} \\
& {[k]_{E}=D a b \int_{-1}^{1} \int_{-1}^{1}[B][C][B] d \xi d \eta}
\end{aligned}
$$

Where, $D$ is the flexural rigidity, $[B]$ the bending stiffness of the element and $[C]$

$$
[B]=\left[\begin{array}{l}
\frac{1}{a^{2}} \frac{\partial^{2}}{\partial \xi^{2}} \\
\frac{1}{b^{2}} \frac{\partial^{2}}{\partial \eta^{2}} \\
\frac{2}{a b} \frac{\partial^{2}}{\partial \xi \partial \eta}
\end{array}\right][N(\xi, \eta)],[C]=\left[\begin{array}{ccc}
1 & v & 0 \\
v & 1 & 0 \\
0 & 0 & (1-v) / 2
\end{array}\right]
$$

Total number of elements in plate is $N_{E}=M * N$,

Total kinetic energy $(T)$ and strain energy $\left(U_{E}\right)$ are

$$
\begin{aligned}
& T=\sum_{E=1}^{N_{E}} \frac{1}{2}\{\dot{u}\}_{E}^{T}[m]_{E}\{\dot{u}\}_{E}=\frac{1}{2}\{\dot{q}\}^{T}[M]\{\dot{q}\} \\
& U_{E}=\sum_{E=1}^{N_{E}} \frac{1}{2}\{u\}_{E}^{T}[k]_{E}\{u\}_{E}=\frac{1}{2}\{q\}^{T}[K]\{q\}
\end{aligned}
$$

Where $\{q\}$ is a vector consisting of all the independent nodal displacements $\left(w, \theta_{x}, \theta_{y}\right)$ and $[M] \&[K]$ are the global mass and stiffness matrices that are assembled from the element mass $[m]_{E}$ and stiffness $[k]_{E}$ matrices. Applying the Lagrange's approach 
$\frac{d}{d t}\left(\frac{\partial T}{\partial\{\dot{q}\})}\right) \frac{\partial U}{\partial\{q\}}=0$

The second order governing differential equation is

$[M]\{\ddot{q}\}+[K]\{\dot{q}\}=0$

This is the discretized model of free vibration of plates

$\{q\}=\{x\} \sin \omega t$

Putting the values of $\ddot{q}, \dot{q}$ in equation

$\omega^{2}[M]\{x\}=[K]\{x\}$

The natural frequency of the system $(w)$ and the associated mode shapes are denoted by $x$. The natural frequencies or the Eigen values are the roots of the characteristics equation

$\left|\left(-\omega^{2}[M]+[K]\right)\right|=0$

Material properties and dimensions for plate

\begin{tabular}{|l|l|}
\hline Parameter & Plate \\
\hline Length (L) & $160 / 1000$ \\
\hline Breadth (B) & $160 / 1000$ \\
\hline Height (H) & $0.6 / 1000$ \\
\hline Density (rho) & 7800 \\
\hline Modulus of Elastisity (E) & 207 Gpa \\
\hline Modulus of Rigidity (v) & 0.3 \\
\hline
\end{tabular}

First three natural frequencies for different mesh sizes for a plate

\begin{tabular}{|l|l|l|l|}
\hline Mesh Size & $6 X 6$ & $7 X 7$ & $8 X 8$ \\
\hline Freq 1 & 8.94896 & 8.93994 & 8.93398 \\
\hline Freq 2 & 14.9334 & 14.9334 & 14.9334 \\
\hline Freq 3 & 33.5536 & 33.661 & 33.7338 \\
\hline & & & \\
\hline Mesh Size & $9 X 9$ & $10 X 10$ & $11 X 11$ \\
\hline Freq 1 & 8.92984 & 8.92685 & 8.92463 \\
\hline Freq 2 & 14.9334 & 14.9333 & 14.9332 \\
\hline Freq 3 & 33.7851 & 33.8226 & 33.8507 \\
\hline
\end{tabular}

\section{INDENTATIONS AND EQUATIONS}

\subsection{Analytically by Rayleigh Method}

The deflection function is the product of beam functions

$W(x, y)=X(x) Y(y)$

Where $X(x)$ and $Y(y)$ are chosen as the fundamental mode shapes of beam having the boundary conditions of plate 
Frequency parameters are defined in terms of $\lambda=w a^{2} \sqrt{\frac{\rho}{D}}$ where a is length dimension which is independent to $v$ but as $D$ depends upon $v$ so do frequency.

For simple supported plate supported at $x=0 \& x=a$

$X(x)=\sin \left(\frac{(m-1) \pi x}{a}\right)$

$(m=2,3,4, \ldots)$

And, free at $y=0 \& y=a$

$Y(y)=1$

$(n=0)$

$Y(y)=1-\frac{2 y}{b}$

$(n=1)$

$Y(y)=\cos \gamma_{1}\left(\frac{y}{b}-\frac{1}{2}\right)-\frac{\sin \left(\frac{\gamma_{1}}{2}\right)}{\sinh \left(\frac{\gamma_{1}}{2}\right)} \cosh \left(\frac{y}{b}-\frac{1}{2}\right) \quad(n=2,4,6, \ldots)$

The value of $\gamma_{1}$ are obtained as the roots of

$$
\begin{aligned}
& \tan \left(\frac{\gamma_{1}}{2}\right)+\tanh \left(\frac{\gamma_{1}}{2}\right)=0 \\
& Y(y)=\sin \gamma_{2}\left(\frac{y}{b}-\frac{1}{2}\right)+\frac{\sin \left(\frac{\gamma_{2}}{2}\right)}{\sinh \left(\frac{\gamma_{2}}{2}\right)} \sinh \left(\frac{y}{b}-\frac{1}{2}\right) \quad(n=3,5,7, \ldots)
\end{aligned}
$$

The value of $\gamma_{2}$ are obtained as the roots of

$\tan \left(\frac{\gamma_{2}}{2}\right)-\tanh \left(\frac{\gamma_{2}}{2}\right)=0$

The indicators $m \& n$ are the number of nodal lines lying in $x-\& y$-directions, respectively including the boundaries as nodal lines, except when the boundary is free

Solving, deflection function or mode shape is $\sin \frac{\pi x}{a}$

The associated natural frequency as

$w^{2}=\frac{\pi^{4} D K}{a^{4} \rho N}$

And fluxral rigidity $D$ is given by

$D=\frac{E h^{3}}{12\left(1-v^{2}\right)}$

Frequency response for first three mode shapes

\begin{tabular}{r|l} 
Mode & Freq \\
\hline 1 & 8.926854 \\
\hline 2 & 14.93329 \\
\hline 3 & 33.82255 \\
\hline
\end{tabular}

www.iosrjournals.org 


\subsection{Experimental values}

The experimental values of frequency parameter in "Stress, Strain and Structural Dynamics- An interactive handbook of formulas, solutions and Matlab Toolboxes"

Frequency response for first three mode shapes

\begin{tabular}{|r|c|}
\hline Mode & Freq \\
\hline 1 & 8.6325 \\
\hline 2 & 14.4408 \\
\hline 3 & 32.7073 \\
\hline
\end{tabular}

Comparing first three natural frequencies conclude is drawn that the best mesh size is 10X10 and further piezo-electric patch is placed on the plate at all position in 10X10 mesh and further the global stiffness and mass matrices are calculated considering the effect of smart material used in the form of patches and state space formulation is done to find out the settling time for each patch.

Finite element formulation and first three modes of vibrations of plate

\begin{tabular}{|l|l|l|l|l|l|l|l|l|l|l|}
\hline 10 & 20 & 30 & 40 & 50 & 60 & 70 & 80 & 90 & 100 \\
\hline 9 & 19 & 29 & 39 & 49 & 59 & 69 & 79 & 89 & 99 \\
\hline 8 & 18 & 28 & 38 & 48 & 58 & 68 & 78 & 88 & 98 \\
\hline 7 & 17 & 27 & 37 & 47 & 57 & 67 & 77 & 87 & 97 \\
\hline 6 & 16 & 26 & 36 & 46 & 56 & 66 & 76 & 86 & 96 \\
\hline 5 & 15 & 25 & 35 & 45 & 55 & 65 & 75 & 85 & 95 \\
\hline 4 & 14 & 24 & 34 & 44 & 54 & 64 & 74 & 84 & 94 \\
\hline 3 & 13 & 23 & 33 & 43 & 53 & 63 & 73 & 83 & 93 \\
\hline 2 & 12 & 22 & 32 & 42 & 52 & 62 & 72 & 82 & 92 \\
\hline 1 & 11 & 21 & 31 & 41 & 51 & 61 & 71 & 81 & 91 \\
\hline
\end{tabular}

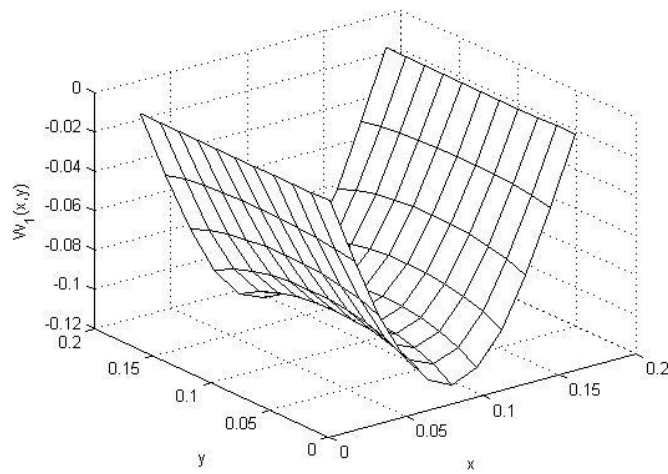

$[m]_{E}=\rho a b \int_{-1}^{1} \int_{-1}^{1}[N(\xi, \eta)]^{T}[N(\xi, \eta)] d \xi d \eta+\ldots$

$\ldots 2 * \rho_{p} a b \int_{-1}^{1} \int_{-1}^{1}[N(\xi, \eta)][N(\xi, \eta)] d \xi d \eta$

$[k]_{E}=\operatorname{Dab} \int_{-1}^{1} \int_{-1}^{1}[B][C][B] d \xi d \eta+\ldots$

$\ldots 2 * D_{p} a b \int_{-1}^{1} \int_{-1}^{1}[B][C][B] d \xi d \eta$ 

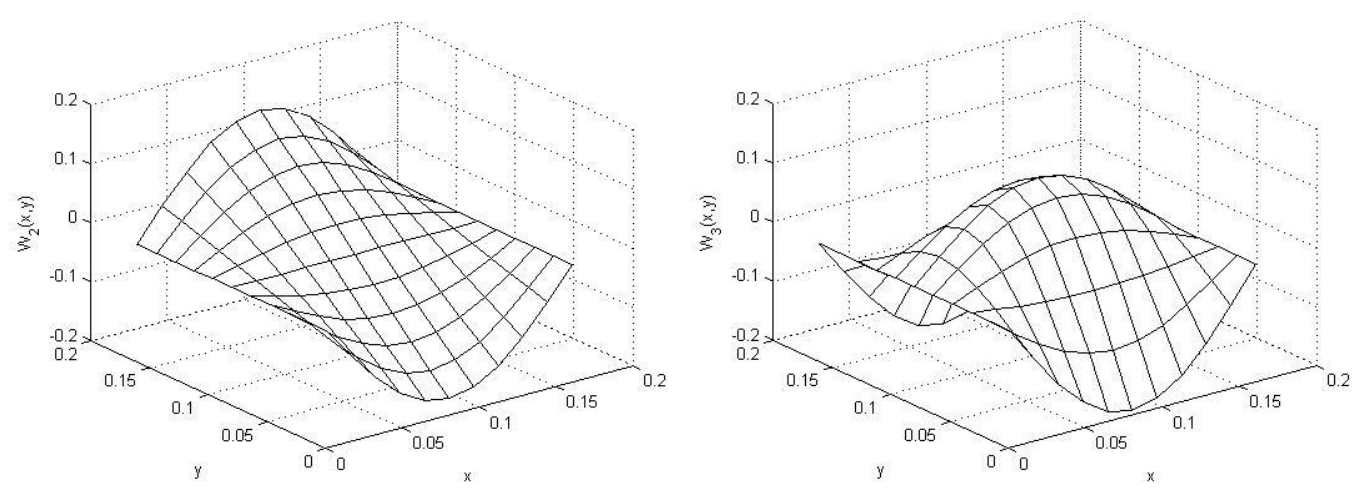

Material Properties and dimensions of Piezo-Patch

\begin{tabular}{|l|l|}
\hline Parameter & Piezo-Patch \\
\hline Length (L) & 0.02 \\
\hline Breadth (B) & 0.02 \\
\hline Height (H) & $1.06 / 1000$ \\
\hline Density (rho) & 7500 \\
\hline Modulus of Elastisity (E) & $63 \mathrm{Gpa}$ \\
\hline Modulus of Rigidity (v) & 0.3 \\
\hline
\end{tabular}

\subsection{Piezoelectric constitutive equations}

According to IEEE standards, in an unstressed 1D dielectric medium if $\varepsilon$ is the dielectric constant for material, the electric displacement $D$ is related to electric field $E$

$D=\varepsilon E$

Similarly, in 1D elastic body placed in a zero electric field if $s$ is the compliance of material (inverse of young's modulus of elasticity) strain $S$ and stress $T$ are related by

$S=s T$

The electric and mechanical constitutive equations for a piezoelectric material are coupled by

$S=s^{E} T+d E$

$D=d T+\varepsilon^{T} E$

\subsection{Piezo patch bonded as sensor}

$D=e S$

The charge per unit area and strain are related by

$D=-E_{p} d_{31} \varepsilon_{11}$

The control voltage produced is, $V_{o}(t)=\frac{-Q}{C_{f}}=\frac{E_{p} d_{31} h_{p}}{C_{f}}\left[\omega^{\prime}(b)-\omega^{\prime}(a)\right]$

Thus sensor output is proportional to the difference of slopes at the extremities of the piezo strip.

3.5 Piezo patch bonded as an actuator

The resisting stress produced is

$\sigma_{11}=E_{p} \varepsilon_{11}-\mathrm{e}_{31} \frac{V}{h_{p}}$

$h_{p}$ is the height of piezo-patch bonded as an actuator

\subsection{State space analysis}




\subsubsection{Model Reduction}

The final equation after embedding the piezoelectric sensor and actuator to the plate, when external force is taken as unit impulse force, is

$$
M \ddot{p}+K p=q_{\text {control }}
$$

$M, K, q_{\text {control }}$ are the mass, stiffness and the force co-efficient vectors of plate. The structure is modelled to retain large number of degrees of freedoms for better accuracy. In active vibration control of flexible structures, however the use of smaller order model has computational advantages. Therefore, it is necessary to apply a model reduction technique to the state space representation. The reduced order system model extraction techniques solve the problem of the complexity by keeping the essential properties of the full model only. The frequency range is selected to span first two or three frequencies of the smart plate in order to find the reduced order model of the system. Its state space mathematical model in principal coordinate system is obtained in Matlab from the modal analysis results. Consider a generalized co-ordinate for reduction as

$p=V z$

$V$ is the modal vectors corresponding to the first two Eigen values. These are the first two columns in the modal matrix.

$$
\begin{aligned}
& M V \ddot{z}+K V z=q_{\text {control }} \\
& V^{T} M V \ddot{z}+V^{T} K V z=V^{T} q_{\text {control }} \\
& M_{\text {red }}=V^{T} M V \\
& K_{\text {red }}=V^{T} K V \\
& F_{\text {red }}=V^{T} q_{\text {control }} \\
& M_{\text {red }} \ddot{z}+K_{\text {red }} z=F_{\text {red }} \\
& \ddot{z}=\left[\begin{array}{l}
\ddot{\omega} \\
\ddot{\theta}_{x} \\
\ddot{\theta}_{y}
\end{array}\right], z=\left[\begin{array}{l}
\omega \\
\theta_{x} \\
\theta_{y}
\end{array}\right]
\end{aligned}
$$

\subsubsection{State space formulation}

In state space formulation, the second order differential equations are converted to first order differential equations. Considering the following notations,

$$
\begin{aligned}
& \omega=x_{1}, \theta_{x}=x_{2}, \theta_{y}=x_{3} ; \\
& \dot{\omega}=\dot{x}_{1}=x_{4}, \\
& \dot{\theta}_{x}=\dot{x}_{2}=x_{5}, \\
& \dot{\theta}_{y}=\dot{x}_{3}=x_{6} ; \\
& \ddot{\omega}=\dot{x}_{4}, \ddot{\theta}_{x}=\dot{x}_{5}, \ddot{\theta}_{y}=\dot{x}_{6} \\
& M_{r e d}\left[\begin{array}{c}
\dot{x}_{4} \\
\dot{x}_{5} \\
\dot{x}_{6}
\end{array}\right]+K_{r e d}\left[\begin{array}{c}
x_{1} \\
x_{2} \\
x_{3}
\end{array}\right]=F_{r e d}
\end{aligned}
$$

Multiply both sides by $M_{r e d}^{-1}$ 
$M_{r e d}^{-1} M_{r e d}\left[\begin{array}{c}\dot{x}_{4} \\ \dot{x}_{5} \\ \dot{x}_{6}\end{array}\right]+M_{r e d}^{-1} K_{r e d}\left[\begin{array}{c}x_{1} \\ x_{2} \\ x_{3}\end{array}\right]=M_{r e d}^{-1} F_{r e d}$

$\left[\begin{array}{l}\dot{x}_{1} \\ \dot{x}_{2} \\ \dot{x}_{3} \\ \dot{x}_{4} \\ \dot{x}_{5} \\ \dot{x}_{6}\end{array}\right]=\left[\begin{array}{ll}0 & I \\ -M_{\text {red }}{ }^{-1} K_{r e d} & 0\end{array}\right]\left[\begin{array}{l}x_{1} \\ x_{2} \\ x_{3} \\ x_{4} \\ x_{5} \\ x_{6}\end{array}\right]+\left[\begin{array}{l}0 \\ M_{r e d}{ }^{-1} V^{T} h\end{array}\right] \omega(t)$

$X=A x(t)+B w(t)$

$Y=C x(t)+D w(t)$

$A$ is known as the system matrix, $B$ is input matrix, $C$ is the output matrix, $D$ is the direct transmission matrix and $w(t)$ is the smallest input force.

$Y=V^{s}(t)=g^{T}[\dot{p}]=g^{T} V \dot{z}$

$Y=g^{T} V\left[\begin{array}{l}x_{4} \\ x_{5} \\ x_{6}\end{array}\right]=\left[0 g^{T} V\right]\left[\begin{array}{l}x_{1} \\ x_{2} \\ x_{3} \\ x_{4} \\ x_{5} \\ x_{6}\end{array}\right]$

Hence,

$$
A=\left[\begin{array}{ll}
0 & I \\
-M_{r e d}{ }^{-1} K_{r e d} & 0
\end{array}\right], B=\left[\begin{array}{l}
0 \\
M_{r e d}^{-1} V^{T} h
\end{array}\right],
$$

$C=\left[\begin{array}{ll}0 & g^{T} V\end{array}\right], D=$ null

\section{Control laws}

In the present work one classical control law, which is based on output feedback and one optimal control law based on state/output feedback is considered. Linear quadratic regulator (LQR) optimal control theory is used to determine the active control gain. The following quadratic cost function is minimized

$j=\frac{1}{2} \int_{0}^{\infty}\left(x^{T} Q x+u^{T} R u\right) d t$

$Q \& R$ represent weights on the different states and control channels and their elements are selected to provide suitable performance. They are the main design parameters. $\mathrm{J}$ represents the weighted sum of energy of the state and control. Assuming full state feedback, the control law is given by

$u=-K x$

with constant control gain

$K=R^{-1} B^{T} P$

Matrix $P$ can be obtained by the solution of the Riccati equation, given by

www.iosrjournals.org 
$A^{T} P+P A+Q-P B R^{-1} B^{T} P=0$

The closed loop system dynamics with state feedback control is given by $\dot{x}=(A-B K) x+\operatorname{Er}(t)$

Alternatively, output feedback control provides a more meaningful design approach in practice. Measured outputs $y$ from sensors are directly feed back to actuators through

$u=-K y$

$\dot{x}=(A-B K C) x+\operatorname{Er}(t)$

$A_{c}=A-B K C$

The quadratic cost function can be written as

$j=\frac{1}{2} \int_{0}^{\infty}\left[x^{T}\left(Q x+C^{T} K^{T} R K C\right) x\right] d t$

\section{POF controller}

Here the minimum settling time is for the patches at $25,26,75$ and $76^{\text {th }}$ position in $10 \mathrm{X} 10$ mesh as shown in the graph between settling time and patch location.
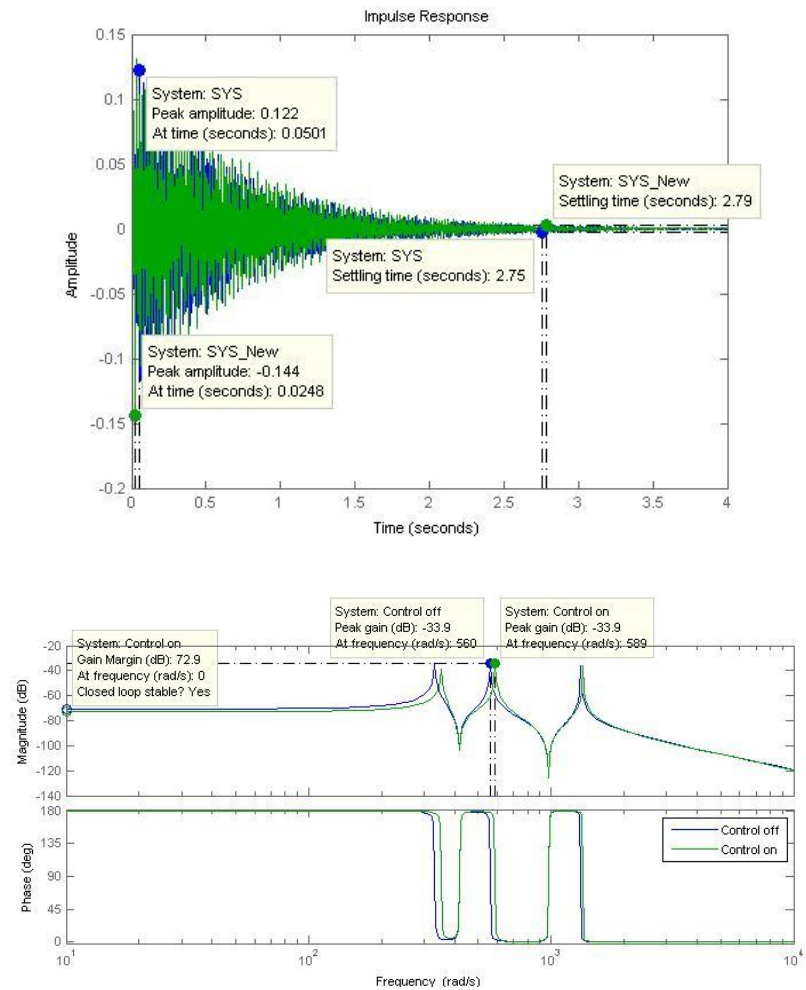

\section{LQR controller}

Here the minimum settling time is for the patches at 25, 26, 75 and $\mathbf{7 6}$ positions for LQR controller in 10X10 mesh as shown in the graph between settling time and patch location.

control off vs direct proportional feedback vs LQR controller 


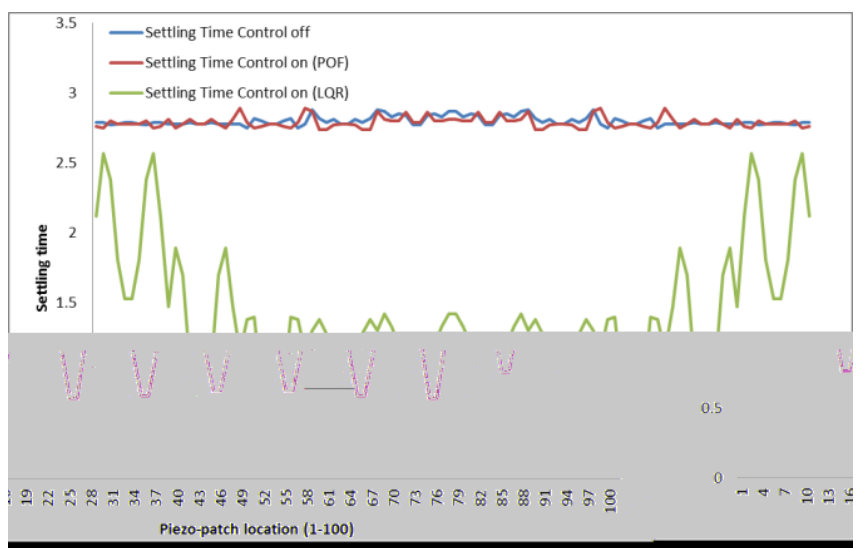

The optimal locations of sensor actuator pair (shown at 25)

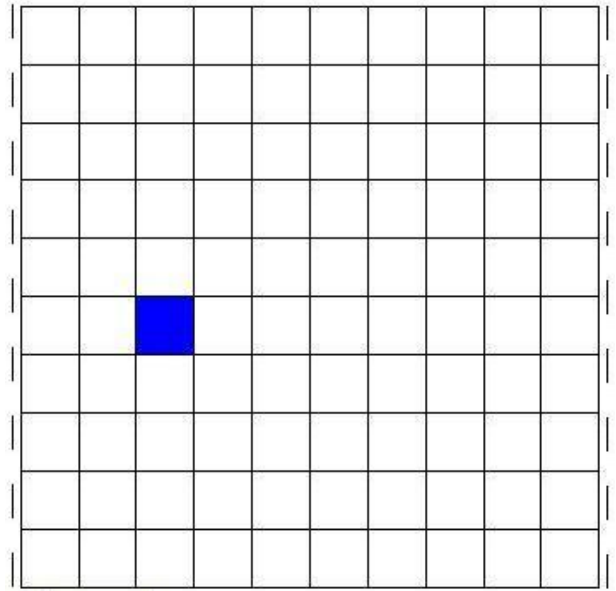

$\mathrm{LQR}$ control law is used to find the optimal location of sensor actuator pair attached in the form of piezo patch, hence the impulse response using LQR controller isTip displacement response and frequency response for control off and control on ( $\mathrm{LQR}$ having $\mathrm{Q}=10^{3}, \mathrm{R}=1$ )

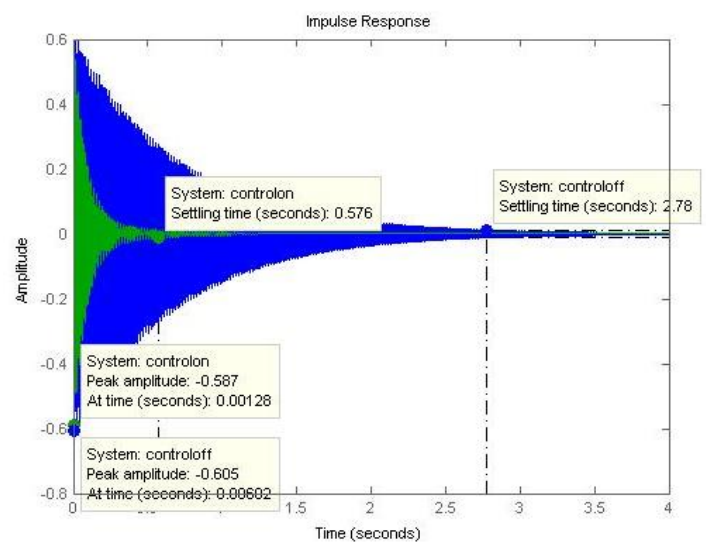




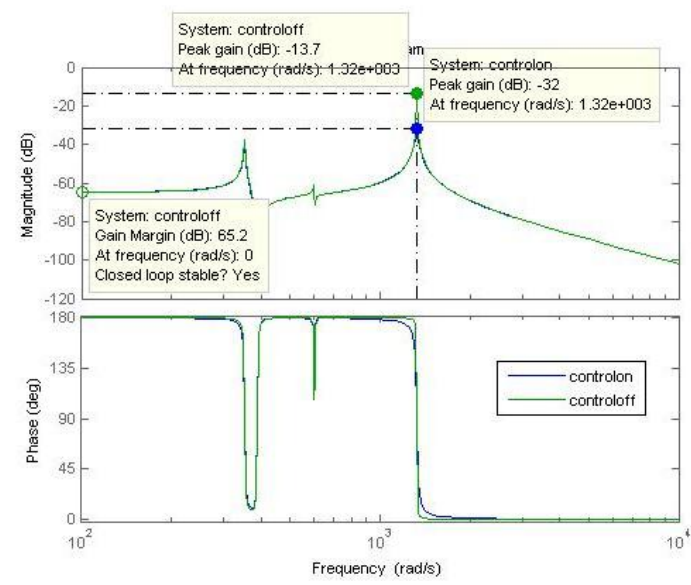

Hence The optimal locations are $\mathbf{2 5 , 2 6 , 7 5}$ and $\mathbf{7 6}$ and the optimal size for patch for the plate having size $(0.16 \times 0.16)$ and mesh size $(10 \times 10)$ our patch size comes out to be $\mathbf{( 0 . 0 2 \times 0 . 0 2 )}$

\section{CONCLUSion}

This research manuscript shows the basic technique of analysis of simple supported plate for Active Vibration Control using piezoelectric sensors and actuators. The optimal location and size of sensor actuator pair for a simple supported plateand control effectiveness of POF and LQR controller is obtained.The numeric example demonstrated the ability of developed method for optimal placement and size of sensor actuator pair based on LQR control strategy. Results concluded that the sensor actuator pair is optimally located based on the lower settling time criteria. It is noted that the control effectiveness of POF controller is insignificant when compares to the LQR controller's. Study also revealed that LQR controller offers optimal effectiveness with lower peaks in settling time as compared to other classical control strategies.

\section{REFERENCES}

[1] Lam K. Y. et al., 1997, “A finite-element model for piezoelectric composite laminates”, Smart Mater. Struct. , 6: 583-591.

[2] Pai P. F. et al., 1998, "Structural vibration control using PZT patches and non-linear phenomena",Journal of Sound and Vibration, 215(2): 273-296.

[3] Balamurugan V. and Narayanan S., 2001, "Shell finite element for smart piezoelectric composite plate/shell structures and its application to the study of active vibration control", Finite Elements in Analysis and Design, 37:713-738.

[4] Li Y. et al., 2001, "Simultaneous optimization of piezoelectric actuator placement and feedback for vibration suppression", PII: S0094-5765(01)00185-0.

[5] Tylikowski A., 2001, "Control of circular plate vibrations via piezoelectric actuators shunted with a capacitive circuit", thinWalled Structures, 39: 83-94.

[6] Mukherjee A. et al., 2002, “Active vibration control of piezolaminated stiffened plates”, Composite Structures, 55: 435-443.

[7] Yaman Y. et al., 2002, “Active vibration control of a smart plate”, ICAS2002 CONGRESS.

[8] Caruso G. et al., 2003, "Active vibration control of an elastic plate using multiple piezoelectric sensors and actuators", Simulation Modeling Practice and Theory, 11: 403-419.

[9] Li Y.Y. et al., 2003, "Modeling and vibration control of a plate coupled with piezoelectric material”, Composite Structures, 62: $155-162$.

[10] Narayanan S. and Balamurugan V., 2003, "Finite element modeling of piezolaminated smart structures for active vibration control with distributed sensors and actuators", Journal of Sound and Vibration, 262: 529-562.

[11] Shimon P. et al., 2005, "Theoretical and experimental study of efficient control of vibrations in a clamped square plate", Journal of Sound and Vibration, 282:453-473.

[12] Luo Q. and Tong L., 2006, "High precision shape control of plates using orthotropic piezoelectric actuators", Finite Elements in Analysis and Design, 42: 1009 - 1020. 
[13] Yang Y. and Zhang L., 2006, “Optimal excitation of a rectangular plate resting on an elastic foundation by a piezoelectric actuator", Smart Mater. Struct.,15: 1063-1078.

[14] Costa L. et al., 2007, "Modeling and numerical study of actuator and sensor effects for a laminated piezoelectric plate", Computers and Structures, 85: 385-403.

[15] Lin J. C. and Nien M.H., 2007, "Adaptive modeling and shape control of laminated plates using piezoelectric actuators", Journal of Materials Processing Technology, 189: 231-236.

[16] Nguyen Q. et al., 2007, "Evolutionary piezoelectric actuators design optimization for static shape control of smart plates", Comput. Methods Appl. Mech. Engrg., 197: 47-60.

[17] Qiu Z. C. et al., 2007, "Optimal placement and active vibration control for piezoelectric smart flexible cantilever plate", Journal of Sound and Vibration, 301: 521-543.

[18] Kang Z. and Tong L., 2008, "Topology optimization-based distribution design of actuation voltage in static shape control of plates", Computers and Structures, 86: 1885-1893.

[19] Shirazi A. H. N. et al., 2011, “Active vibration control of an FGM rectangular plate using fuzzy logic controllers", Procedia Engineering, 14: 3019-3026.

[20] Yang B., 2005, "Stress, Strain and Structural Dynamics- An interactive handbook of formulas, solutions and Matlab Toolboxes", Elsevier Academic Press

[21] Liessa W., 1990, “Vibration of Plates”, U.S. government printing press

[22] Petyt M., 1998, "Introduction to finite element vibration Analysis", Cambridge University Press 